\title{
Erratum: Role of inertia for the rotation of a nearly spherical particle in a general linear flow [Phys. Rev. E 91, 053023 (2015)]
}

\author{
F. Candelier, J. Einarsson, F. Lundell, B. Mehlig, and J.-R. Angilella
}

(Received 23 October 2015; published 19 November 2015)

DOI: 10.1103/PhysRevE.92.059901 PACS number(s): 47.15.G-, 83.10.Pp, 47.55.Kf, 47.10.-g, 99.10.Cd

In this paper we investigated the role of inertia for the rotation of a nearly spherical particle in a general linear time-independent flow. As we continued to work on related questions we realized that the argument used in this paper to show that Eq. (7) evaluates to zero is valid for a constant shear flow, but not in general. This implies that our results for the shear flow [Eq. (22)] are correct and consistent with the results of Refs. [1-3]. But for other linear flows Eq. (7) may give rise to additional contributions to the angular particle velocity [Eq. (19)]. As a consequence Eq. (23b) for the case of a purely rotational flow is replaced by $\dot{\theta}=(\epsilon / 30)\left[\mathrm{St}-\mathrm{Re}_{s}\right] \sin 2 \theta$. The right-hand side of this equation evaluates to zero for neutrally buoyant particles $\left(\mathrm{St}=\mathrm{Re}_{s}\right)$. This means that a neutrally buoyant particle rotates precisely like the surrounding fluid as it must since the fluid rotates as a rigid body. For an elongational flow the factor $11 / 70$ in Eq. (24b) is replaced by 8/42. The qualitative conclusions for a nearly spherical particle in an elongational flow do not change.

[1] J. Einarsson, F. Candelier, F. Lundell, J. R. Angilella, and B. Mehlig, Phys. Fluids 27, 063301 (2015).

[2] J. Einarsson, F. Candelier, F. Lundell, J. R. Angilella, and B. Mehlig, Phys. Rev. E 91, 041002(R) (2015).

[3] T. Rosen, J. Einarsson, A. Nordmark, C. Aidun, F. Lundell, and B. Mehlig, arXiv:1508.04976. 